\title{
A comparison of reversal and extradimensional shifts in Jordanian children
}

\author{
MITRI E. SHANAB \\ California State University, Fresno, California 93740
}

and

\author{
ABDALLAH BAKER YASIN \\ University of Jordan, Amman, Jordan
}

\begin{abstract}
Reversal (R) and extradimensional (ED) shifts were studied in a large Jordanian sample which included six age groups $(4,5,6,9,10$, and 11 years). No sex differences were found. Consistent with other findings based on American samples, a highly significant Age by Shift interaction effect was found, reflecting the ease of executing a $R$ relative to an ED shift at a very young age and the reversal of this effect at about 9 years of age. The results provide further support for the Kendlers' two-stage mediational theory.
\end{abstract}

The literature is replete with studies which have shown that, while rats and preschoolers solve an extradimensional (ED) shift faster than a reversal (R) shift, the opposite is true of older children and young adults (Kendler \& Kendler, 1975). Moreover, children of approximately 5 years of age solve both shifts at comparable rates. In explaining these ontogenetic changes in problem-solving behavior, Kendler and Kendler (1962) proposed a dual-stage mediational model, according to which the behavior of rats and very young children is assumed to be under the control of specific external stimuli, whereas the behavior of older children and young adults is not governed by such a singleunit system. Instead, these subjects behave according to a mediational system in that they solve a discrimination task by utilizing conceptual mediational links between the overt response and the external stimulus. The lack of difference in executing either shift at about age 5 is viewed as marking a transition period that reflects a progression from stimulus-bound responding to mediational responding. As Nehrke (1973) has recently suggested, conceptual shift behavior appears to develop from a stage which is dominated by mediationally deficient behavior to one in which mediationally proficient functioning becomes more prevalent.

Initially, the mandatory shift paradigm was used to investigate problem-solving behavior as reflected in discrimination learning in both humans and animals. According to this design, subjects are required to learn

This research was carried out by the second author in partial fulfillment of his master's degree under the direction of the first author, who was on sabbatical leave at the University of Jordan, Department of Psychology, Amman, Jordan, during the 19751976 academic year. Reprint requests should be sent to Mitri E. Shanab, Department of Psychology, California State University, Fresno, California 93740. to discriminate first between two stimuli that differ on two dimensions, with one dimension being arbitrarily designated as correct and the other as incorrect; then a shift in conditions is introduced (see Figure 1). In a $\mathrm{R}$ shift, the previously correct dimension continues to be correct but the previously correct cue in that dimension becomes incorrect. In an ED shift, the previously correct dimension becomes irrelevant and the previously

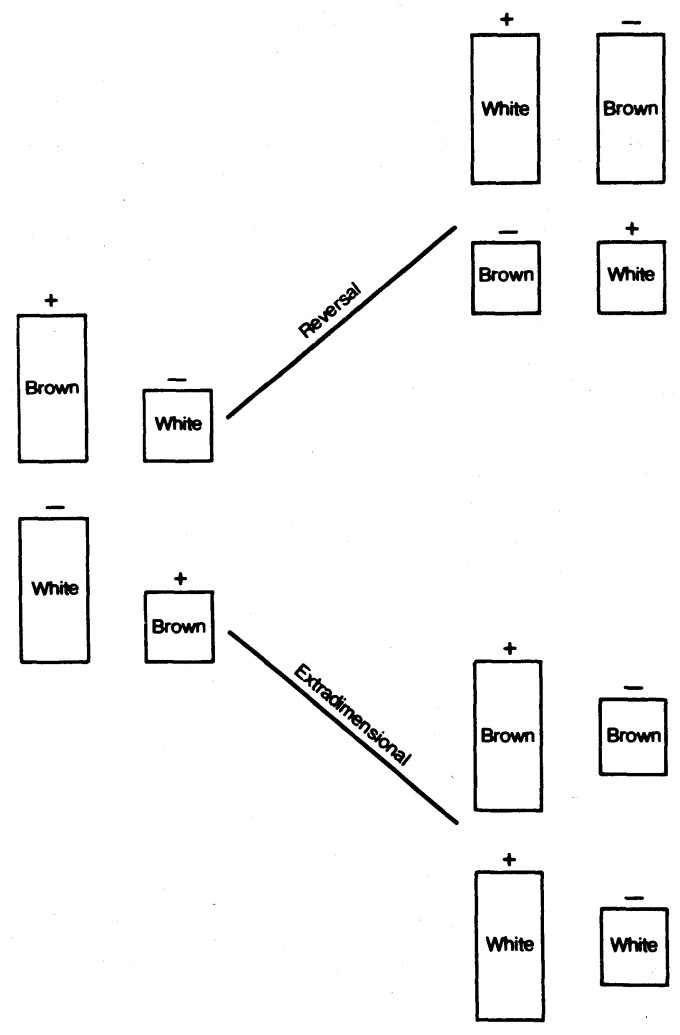

Figure 1. Illustration of the R-ED design. 
incorrect dimension becomes correct. The mandatory design is methodologically vulnerable in that the superiority of the $\mathrm{R}$ over the ED shift, among older children and college students in particular, could be attributed to the fact that these subjects could have been adventitiously reinforced during the ED shift for responding in the same manner as they did in the preshift phase. To control for such fortuitous intermittent reinforcement, Buss (1956) and Kendler and D'Amato (1955) re-paired the original stimuli in the shift phase such that each pair differed in terms of one dimension only. Both studies showed that college students solved the $\mathrm{R}$ shift faster than the ED shift, thus providing further support to the mediational interpretation. Several other procedures have since been developed to control for adventitious intermittent reinforcement (cf. Kendler \& Kendler, 1975; Slamecka, 1968).

The vast majority of studies which investigated conceptual shift behavior are based on U.S. samples. No known studies have been carried out in non-European derived cultures. Thus, the main purpose of the present study was to test the generality of the ontogenetic trend in shift behavior in a Middle Eastern culture which is distinctly different from the American culture. The mandatory paradigm was used as a preliminary procedure in a series of cross-cultural investigations of this phenomenon.

\section{METHOD}

\section{Design}

A two-phase mandatory shift study was used sampling six age groups: $4,5,6,9,10$, and 11 years. In Phase 1 , each subject learned to choose the correct member of a pair of stimuli that differed on two dimensions: size (either large or small) or color (either brown or white). Following criterion learning, a random half of the subjects was given a $R$ shift in which the previously relevant dimension remained the same, while the other half received an ED shift in which the irrelevant dimension became relevant. Thus, in Phase 2 a 2 by 2 by 6 factorial design was used combining sex, two types of shift, and six age levels.

\section{Subjects}

A total of 288 Jordanian subjects, 12 per group, participated in the study. The combined mean age for the males and females in the six age groups was: $4.5,5.3,6.7,9.6,10.6$, and 11.6 years. The subjects were selected from several nursery and public schools in Amman, Jordan. Although random assignment was observed, the subjects were matched within each age group in terms of scholastic achievement as determined by grades or teachers' evaluations.

\section{Apparatus}

The apparatus was a lacquer-painted turntable made of 2 -cm-thick plywood with a $25-\mathrm{cm}$ radius. The turntable, which was mounted on a $15-\mathrm{cm}$ wooden base, was divided in half by a perpendicular $18 \times 48 \mathrm{~cm}$ plywood board which served to screen the actions of the experimenter. On one of the two halves of the turntable, two depressions were made such that each could hold a marble. The depressions were $8 \mathrm{~cm}$ apart and $8 \mathrm{~cm}$ from the perpendicular board. The discriminanda were four wooden objects that differed on two dimensions, either size (large or small) or color (white or brown). Each stimulus object had a tiny depression in its base so that it could be easily placed over the top of the marble. On both sides of the apparatus were placed two wooden boards with 50 holes in each to hold the marbles won by the subjects.

\section{Procedure}

Each subjects was tested individually. The subject sat facing the experimenter and the apparatus which was placed on a small table. As soon as the subject was comfortably seated, the experimenter read the following instructions, which were a close translation of the instructions used by Kendler and Kendler (1959).

"This is the game we are going to play, (child's name). Before we start, listen carefully and I will tell you how the game is played. See, there are two things here. When we start the game you will choose one of them and pick it up. If you are right you will find a marble under it. If you are wrong then you won't find anything under it. Each time you may choose only one. Then I will turn it around, like this, and then you may choose only one. The game is to see how soon you can get a marble every time you choose. If you get the marble you put it into one of these holes. When we finish the game you may choose one of the prizes over there." The experimenter then pointed to the prizes which were placed about $1 \mathrm{~m}$ to the side of the experimenter. The prizes were placed in little nylon bags which consisted of either hard candy, toffe, five pencils, peanuts, or small chewing gum packages. The experimenter then said, "Remember the game is to see how soon you can get a marble every time you choose." This sentence was repeated every 15 trials.

The stimuli were presented one pair at a time and differed on two dimensions simultaneoulsy, size and color. Only one dimension was correct for any given subject (see Figure 1). To control for the reinforced stimulus value, each of the four possible stimulus values was correct for a random quarter of the subjects. To control for sequence effects, the pairs were presented in an $a-b-b-a$ order. In addition, each stimulus in a pair appeared equally of ten on the right and the left.

Initially, each subject was trained to a criterion of nine consecutive correct responses, or was otherwise given 100 trials. Immediately following this, the subjects received either an ED or $\mathrm{R}$ shift according to a prearranged random schedule. For subjects in the $R$ condition, if large was the positive stimulus in the initial discrimination, small became the correct stimulus, while color became the irrelevant dimension. For subjects in the ED condition, if large was the correct stimulus, then color became correct, regardless of size. In both shifts, two pairs of stimuli were presented, one at a time, such that they differed on one dimension only. Thus, for the $\mathrm{R}$ shift, one pair would be large brown and large white, while the second would be small brown and small white. For the ED shift, one pair would be, say large brown vs. small brown, while the other would be large white vs. small white. Counterbalancing for position, order of presentation, and stimulus value was also observed in the shift phase. The criterion of learning a $R$ or ED shift was nine successive correct choices.

\section{RESULTS AND DISCUSSION}

All analyses are based on number of trials to criterion. The results of a 2 (sex) by 2 (type of shift in Phase 2) by 6 (age) analysis of variance test on mean performance during the initial discrimination task yielded nonsignificant main effects (all Fs $<1$ ). All possible interactions with the main effects were similarly not significnat. Except for the Sex by Age interaction effect $[F(5,264)=1.32, \mathrm{p}>.05]$, the interactions yielded $F$ values less than 1 . This complete lack of differences in Phase 1 attests to the appropriateness of the randomiza- 
Table 1

Mean Number of Trials to Criterion During the Shift Discrimination Phase

\begin{tabular}{ccc}
\hline Age in Years & Reversal & Extradimensional \\
\hline 4 & 62.41 & 27.87 \\
5 & 41.37 & 30.29 \\
6 & 32.20 & 33.00 \\
9 & 21.25 & 35.37 \\
10 & 20.58 & 27.20 \\
11 & 19.20 & 29.83 \\
\hline
\end{tabular}

tion procedure, for apparently all subjects were equivalent on the initial discrimination regardless of their sex, age, or type of shift received in Phase 2. The results of the shift discrimination of Phase 2 were subjected to the same test, which yielded a significant age effect $[\mathrm{F}(5,264)=5.35, \mathrm{p}<.001]$, reflecting faster learning with increased age. However, no significant effects due to either type of shift or sex (both Fs $<1$ ) were found. Similarly, neither the triple interaction $[\mathrm{F}(5,264)=1.63$, $p>.05]$ nor the two double interactions of shift with sex and sex with age were significant (both $F s<1$ ). However, the Age by Shift interaction effect was highly significant $[F(5,264)=6.82, p<.001]$. As Table 1 shows, there is a consistent increase in the ease of executing a $R$ shift as age increases, but no consistent trend appears to be associated with the ED shift. It can also be seen that, while very young children apparently solve an ED shift faster than a $\mathrm{R}$ shift, the opposite seems to be true of children 9 years and older. Moreover, Table 1 clearly shows that 6-year-old children execute both shifts at comparable rates, probably re-

flecting a transition period comparable to the one observed in the American samples. These results are obviously supportive of the ontogenetic trend previously reported in studies based on American samples (Kendler $\&$ Kendler, 1975). Thus, despite the diversity between the American and Jordanian cultures, conceptual development, as measured by the R-ED paradigm, in both cultures seems to be governed by the same principles.

\section{REFERENCES}

Buss, A. H. Reversal and nonreversal shifts in concept formation with partial reinforcement eliminated. Journal of Experimental Psychology, 1956, 52, 162-166.

KendleR, H. H., \& D'Amato, M. R. A comparison of reversal shifts and nonreversal shifts in human concept formation behavior. Journal of Experimental Psychology, 1955, 49, $165-174$.

KENDLER, H. H., \& KendLeR, T. S. Vertical and horizontal processes in problem solving. Psychological Review, 1962, 69, 1-16.

KendleR, H. H., \& KendleR, T. S. From discrimination learning to cognitive development: A neobehavioristic odyssey. In W. K. Estes (Ed.), Handbook of learning and cognitive processes (Vol. 1). Hillsdale, N.J: Erlbaum, 1975 Pp. 191-247.

Kendler, T. S., \& Kendler, H. H. Reversal and nonreversal shifts in kindergarten children. Journal of Experimental Psychology, 1959, 58, 56-60.

NeHRKE, M. F. Age and sex differences in discrimination learning and transfer of learning. Journal of Gerontology, $1973,28,320-327$.

Slamecra, N. J. A methodological analysis of shift paradigms in human discrimination learning. Psychological Bulletin, $1968,69,423-438$.

(Received for publication November 15, 1977.) 